\title{
L'extension sous toutes les latitudes de « la république des lettres »
}

Entretien mené par Julien Jeusette

\section{Blaise Ndala}

\section{(2) OpenEdition}

\section{Journals}

Édition électronique

URL : https://journals.openedition.org/coma/5812

DOI : $10.4000 /$ coma. 5812

ISSN : 2275-1742

Éditeur

Institut des textes \& manuscrits modernes (ITEM)

\section{Référence électronique}

Blaise Ndala, «L'extension sous toutes les latitudes de « la république des lettres » », Continents manuscrits [En ligne], 15|2020, mis en ligne le 15 octobre 2020, consulté le 12 janvier 2023. URL http://journals.openedition.org/coma/5812 ; DOI : https://doi.org/10.4000/coma.5812

Ce document a été généré automatiquement le 12 janvier 2023.

\section{cc)}

Creative Commons - Attribution - Pas d'Utilisation Commerciale - Pas de Modification 4.0 International - CC BY-NC-ND 4.0

https://creativecommons.org/licenses/by-nc-nd/4.0/ 


\title{
L'extension sous toutes les latitudes de « la république des lettres »
}

Entretien mené par Julien Jeusette

\author{
Blaise Ndala
}

\section{NOTE DE L'ÉDITEUR}

BLAISE NDALA est né en 1972 en RDC et il vit depuis 2007 à Ottawa au Canada, où il travaille en tant que juriste au sein de la fonction publique fédérale, après avoir été représentant en Haïti de l'ONG des droits humains Avocats sans frontières Canada. Ses deux romans, J'irai danser sur la tombe de Senghor (L'Interligne, 2015 / Vents d'ailleurs, 2019) et Sans capote ni kalachnikov (Mémoire d'encrier, 2017) ont reçu des prix prestigieux. Le premier a notamment remporté le « Prix du livre d'Ottawa », et le second a remporté l'édition 2019 du concours radiophonique canadien « Le Combat national des livres ». Le réalisateur franco-algérien Rachid Bouchareb a récemment acquis les droits de J'irai danser sur la tombe de Senghor pour l'adapter au cinéma. Régulièrement invité dans les médias à titre de chroniqueur, Blaise Ndala est aujourd'hui considéré comme l'une des grandes voix du roman au Canada francophone. Sa troisième œuvre de fiction, fresque à la fois historique et contemporaine sur le Congo et sa décolonisation, paraîtra en janvier 2021.

JULIEN JEUSETTE : La notion d'espace littéraire « mondial » a-t-elle un sens pour vous?

Blaise Ndala : Passé la seconde où mon esprit questionne la présence dans l'énoncé de l'adjectif « mondial », le seul sens que je lui donnerais renverrait à l'ensemble de la production littéraire versée à la curiosité du lecteur, comme une extension sous toutes les latitudes de «la république des lettres». J'entends par là un espace immatériel qui désignerait non plus, comme sous la Renaissance, un territoire intellectuel réunissant les seuls lettrés européens liés par des "valeurs communes » et par le latin dans lequel furent alors mâtinés création littéraire, échanges épistolaires et rencontres autour des textes, mais cette fois un territoire bien plus 
vaste. J'y vois un territoire tout en contrastes, transcendant la géographie, où le poème arabe écrit à Naplouse fait écho au slam créole livré à Jacmel en Haïti, où une tragédie russe prend corps sous le ciel de Lubumbashi, où un conte en bambara revit dans la langue de Shakespeare à l'ombre d'une résidence pour aînés de Toronto. C'est le lieu de souligner le rôle plus ou moins occulté que jouent en faveur de la création littéraire les traducteurs, si souvent anonymisés, rôle sans lequel un tel espace ne serait qu'une juxtaposition d'imaginaires. Leur art on ne peut plus précieux nous permet, en effet, de fluidifier le dialogue autour de l'universelle condition humaine, d'abolir des frontières qui n'en sont pas.

J. J. : À l'heure où les écrivains d'origine congolaise vivent éparpillés à travers le monde, considérez-vous vous qu'il existe quelque chose comme une "littérature congolaise" aujourd'hui? Un «style» congolais? Faites-vous partie d'un quelconque «réseau» congolais international ?

B. $\mathbf{N}$. : Je fais le constat qu'il existe bel et bien une littérature congolaise comme il existe une littérature belge réunissant sous la même bannière aussi bien une Amélie Nothomb racontant le Japon depuis les bords de Seine, que sa compatriote Geneviève Damas qui convoque la Centrafrique depuis le Plat-Pays. Que le colloque «Gobal Congo " qui nous a réunis voilà un an à Milan nous ait conviés à sa tribune, Sinzo Aanza et moi - parmi d'autres -, alors que je vis en Ontario au Canada où je suis publié, et que Aanza vit à Kinshasa où il offre au public l'essentiel de sa production littéraire, est à l'évidence le signe qu'au-delà des lieux d'où nous créons, se dessine une filiation littéraire qui conjugue des éléments aussi épars que l'origine nationale, une certaine idée d'un socle culturel commun, le théâtre où nos imaginaires puisent matière à dire et à interroger le monde; bref, toutes choses que je laisse aux cogitations de ceux dont c'est le métier. Ce que je sais, c'est que mon ancrage dans l'espace littéraire canadien n'est ni monolithique ni exclusif. Dès lors, il ne saurait être utilisé comme motif d'extranéité, tel que cela est observé au fil du débat entourant la question de la nationalité congolaise au sens technique, véritable serpent de mer s'il en est, depuis la chute de Mobutu.

Quant au style, je doute qu'il y en ait un qui soit particulier à un pays, le Congo ou un autre. Au-delà du recours à un idiome commun, je ne crois pas déceler dans le style d'un Michel Houellebecq quoi que ce soit qui se rapproche de l'art du roman que pratique une Marie Darrieussecq, alors que dans le même registre, le style d'un Sony Labou Tansi, en net contraste avec celui de son aîné et compatriote Thicaya U Tam'si, se décline comme une réplique complètement assumée de celui d'un Gabriel Garcia Marquez. Je ne nie donc pas que l'on puisse déceler ici et là des influences, lesquelles déborderont de toute façon du cadre matériel congolais. Mais je ne crois pas qu'il existe au pays de Lomami Tshibamba un style qui, opérant par une sorte de déterminisme, nous gratifierait du sceau d'une «identité » décelable tous genres confondus.

Enfin, sur le fait d'appartenir le cas échéant à un « réseau » congolais international, force est de reconnaître que je n'ai jusqu'ici participé à rien qui eût formellement validé une telle assertion. Je compte néanmoins parmi les auteurs congolais quelques bons amis avec qui je maintiens un contact assez suivi, les nouvelles technologies aidant. Avec certains, il nous est arrivé de tirer des plans sur la comète comme les écrivains savent si bien le faire, mais rien qui ait accouché d'une quelconque structure. 
J. J. : La «World literature » et la sociologie de la littérature pensent souvent le rapport de l'écrivain à l'écriture en termes de «stratégie». Ce terme vous semble-t-il adéquat? Diriezvous que vous avez organisé votre succès (en choisissant telle maison d'édition plutôt qu'une autre, en écrivant de telle manière plutôt que d'une autre, en choisissant une langue de traduction plutôt qu'une autre...) ?

B. N. : N'ayant eu jusqu'ici nul prétexte pour m'intéresser à la question telle que la conçoivent les tenants de la «World literature » et les sociologues de la littérature, je ne peux m'exprimer que du haut de ma modeste expérience. Et il se trouve qu'à la différence de ceux qui ont "pensé » leur carrière d'écrivain avant d'être publiés et pour certains avant de connaître "le succès ", c'est sur le tard que j'ai franchi le Rubicon de la publication. Au départ, faudrait-il le préciser, ce fut pour faire plaisir à quelqu'un qui croyait au potentiel d'un vieux manuscrit qui traînait dans mes tiroirs. Je n'ai rien organisé à proprement parler, ni en 2012 lorsque j'ai, pour la première fois, envoyé un texte à un éditeur dans ma ville, Ottawa, ni à la fin de l'année écoulée lorsque, après deux romans publiés au Canada, un ami m'a suggéré de m'adresser à un éditeur européen - parce que selon lui le manuscrit que je venais de lui faire lire devrait avoir un écho particulier auprès des publics européen et africain. Les livres publiés en Amérique du Nord, je suis le premier à le reconnaître, même avec un bon distributeur comme celui dont j'ai bénéficié chez mon dernier éditeur canadien, ne trouvent pas aussi facilement le chemin des librairies bordelaises ou namuroises, pour ne pas parler des bibliothèques de Kinshasa ou de Kikwit.

Mais cela étant dit, même si j'ai suivi le conseil de mon ami, lui-même un des écrivains africains les plus en vue du côté de Paris, même si je sais que pour le meilleur et pour le pire, la France où mon prochain livre sera publié - ici je ne parle pas de la réédition récente de mon premier roman chez Vents d'ailleurs - reste pour l'écrivain africain à la fois le lieu «idéal» d'édition et celui d'une certaine " légitimation » au sein de l'espace francophone, je ne laisse pas une telle conjoncture brouiller ma vue. Je reste conscient que la France n'a nullement besoin des écrivains des «marges» que nous sommes, Africains, Québécois, Franco-Ontariens, gens des Caraïbes, etc., pour garantir sa place sur l'échiquier littéraire mondial. Ce qui veut dire que l'écrivain canadien d'origine congolaise que je suis commettrait une erreur de mesurer son "empreinte " à l'étalon d'une édition parisienne, plutôt qu'à celui, autrement plus important en ce qui me concerne, d'être lu et abondamment lu au Canada, au Congo et ailleurs dans le monde.

Aurais-je écrit des livres différents, abordé des thèmes autres dans mes écrits, si j'avais été publié depuis Kinshasa ou Port-au-Prince où j'ai séjourné un temps ? S'il m'est difficile de répondre à cette question a posteriori, ce serait faire preuve de déni que de ne pas reconnaître, malgré tout, que la sensibilité de l'écrivain ne puise pas que dans le terreau de sa terre d'origine. Elle est également sujette aux soubresauts que lui renvoie l'environnement dans lequel il baigne, environnement qui influence parfois, avouons-le tout de suite, les fameuses lignes éditoriales à l'aune desquelles tel manuscrit sera refusé à Montréal alors qu'il eût trouvé preneur à Abidjan. Pour ma part, aussi profondément que je sonde mon âme, je ne pense pas avoir «choisi » d'écrire de telle manière plutôt que telle autre, dans le dessein de séduire un lectorat que j'aurais jugé plus " porteur ». À y réfléchir, puisque vous m'en donnez l'occasion, et avec le recul, il me semble qu'aussi bien mes choix thématiques que mon écriture auraient pu ne pas concourir à ce que vous nommez « succès ", puisque mon lectorat, pour le moment, est majoritairement canadien. Or penser «stratégie » eût alors 
suggéré soit que je verse dans un exotisme tropicalisant dans mes textes, soit que je cible des sujets pour lesquels, objectivement, le gros de mon lectorat aurait de l'appétence. Si beaucoup a été dit sur mes romans, je n'ai pas encore été exposé à ce type de "reproche" auquel les écrivains africains dits de la diaspora sont relativement sensibles, certains plus que d'autres. Enfin, pour en venir à la question de la traduction, le passage du français au russe pour ce qui est de mon premier roman ne relève pas d'un choix personnel, mais tout simplement de l'intérêt que «J'irai danser sur la tombe de Senghor » a rencontré de la part des éditeurs torontois et moscovite qui en ont acquis les droits. Difficile de subodorer qu'un Africain vivant à Ottawa veuille organiser son succès littéraire en rêvant d'interminables files d'attente au salon du livre de Saint-Pétersbourg, non?

J. J. : Votre roman Sans capote ni kalachnikov montre à la fois le simplisme et l'ineptie d'une certaine représentation occidentale de l'Afrique. La littérature a-t-elle selon vous un rôle politique à jouer? Vous considérez-vous comme un écrivain engagé ? Comment liez-vous votre engagement intellectuel à l'écriture fictionnelle?

B. N. : La littérature a été, de tout temps, un espace politique, même si je suis le premier à admettre qu'une œuvre littéraire n'a pas besoin de prendre la forme d'un manifeste pour interroger la société dans ses dysfonctionnements, dire la grandeur des petites gens et la petitesse des puissants, la bêtise humaine sous toutes les latitudes. Que je sois un écrivain dont les textes transpirent d'une conscience politique, cela ne fait pas l'ombre d'un doute. Sans fausse humilité aucune, je laisse cependant le manteau et le privilège de l'écrivain engagé à celles et ceux qui, au-delà $\mathrm{du}$ message politique que véhiculent leurs livres, sortent dans l'arène civile et $\mathrm{y}$ posent des actes ayant prise directe avec la transformation de la cité. Là se situe l'engagement politique véritable, lequel ne va d'ailleurs pas sans son lot de vicissitudes. Il suffit d'observer autour de nous pour identifier le profil que j'évoque ici, puisque les concernés ne cachent pas leur intérêt pour la chose politique derrière la simple création littéraire. D’Aimé Césaire à Luis Sepúlveda en passant par Ken Saro Wiwa - pour ne convoquer que des écrivains proches de nous d'un point de vue temporel-, nous pouvons ainsi voir certains militer dans les rangs des partis politiques, au sein de mouvements sociaux dont les agendas sont connus de tous, tandis que d'autres encore multiplient des tribunes pour attirer l'attention sur telle ou telle cause qui épouse leurs convictions : ici, les droits des personnes LGBTQ+, làbas une dictature ou une théocratie obscurantiste à déboulonner, plus loin une forêt et ses écosystèmes à arracher à la voracité du capitalisme sauvage. Force est de reconnaitre que le fonctionnaire que je suis à la ville aurait beaucoup à prouver avant de revendiquer quoi que ce soit de semblable. Tout au plus l'ego de l'écrivain pourrait-il supputer que dans un texte comme "Sans capote ni kalachnikov" puisque vous le nommez -, un mordu de l'engagement politique trouvât deux ou trois « pièces à combustion ». Si tant est que la chose soit déjà arrivée, je n'en suis pas informé.

J. J. : Comment voyez-vous la situation de l'écrivain francophone au Canada? Avez-vous une expérience du milieu littéraire belge, où vous avez habité? Y a-t-il des échanges littéraires entre le Canada et l'Europe, entre le Canada et l'Afrique?

B. N. : La situation de l'écrivain francophone est à l'image de celle de la langue française dans cette partie de l'Amérique du Nord. Une langue enracinée ici depuis plus de 400 ans, qui compte à ce jour un peu plus de 10 millions de locuteurs, et qui n'a pas cessé de tisser sa toile à côté et malgré le rouleau compresseur culturel qu'est 
l'Amérique anglophone. Ce qui frappe l'écrivain francophone venu d'Afrique, c'est à la fois la grande vitalité de la production littéraire en langue française - et pas seulement au Québec, seule communauté francophone d'Amérique du Nord qui évolue en situation de majorité linguistique -, et le peu de passerelles qui existent entre les mondes du livre français et anglais à l'échelle du Canada. Illustration parmi tant d'autres des «deux solitudes» maintes fois décrites par les Canadiens, le faible nombre de traductions d'œuvres littéraires d'une langue officielle à l'autre, y compris en incluant les bestsellers salués hors des frontières du pays. Pareillement, le nombre tout aussi insignifiant d'événements littéraires mettant en dialogue écrivains francophones de l'Ontario français et du Québec, à leurs pairs de la ColombieBritannique et de l'Alberta, pour ne citer que ces provinces parmi les plus peuplées du Canada. Si vous y ajoutez le peu de place qu'occupent dans les programmes d'enseignement et dans les instances de légitimation des écrivains issus des Premières nations, vous obtenez non plus deux, mais « trois solitudes » à la grandeur de la fédération. Je vois certes les choses bouger petit à petit, mais il s'agit d'un mouvement extrêmement lent qui ne portera pas de fruits avant au moins plusieurs décennies encore. Sur une note plus réjouissante, comme écrivain, je ne suis pas indifférent au fait que le Canada d'expression française, même s'il appartient à la marge, a fini au fil du temps par se constituer en un pôle littéraire majeur au sein de la Francophonie, en particulier à partir du Québec. Si Paris continue à jouir de son aura de "centre», le dynamisme du fait littéraire français est tel au pays de Kim Thúy et de Patrice Desbiens, que l'écrivain d'ici trouve d'un océan à l'autre, entre éditeurs prestigieux et programmes de soutien à la création, un espace qui a de quoi faire des envieux sous d'autres latitudes, et pas que dans les pays les moins nantis. De cela, nous pouvons nous féliciter.

Je n'ai pas d'expérience du milieu littéraire belge, puisque je n'ai rien publié du temps où je vivais en Belgique, même si j'y ai écrit au moins un roman qui n'a d'ailleurs jamais quitté le fond de la valise que j'ai traînée de Bruxelles à Ottawa en passant par Montréal.

Des échanges littéraires entre le Canada et l'Europe, je pense qu'il y en a toujours eu, sous différentes formes. Que l'on parle de coédition ou d'événements littéraires comme les salons du livre, des programmes de bourses et de résidences d'écritures, des colloques, des Prix littéraires transatlantiques ou autres jumelages d'auteurs au niveau des régions ou des municipalités, il existe une longue tradition à laquelle j'ai pu moi-même m'associer depuis la sortie en 2014 de mon premier roman. La même chose doit avoir cours du côté anglophone, même si je suis peu au fait de ce qui s'y passe, aucun de mes deux livres n'ayant encore été traduit dans la langue de Margaret Atwood. En parallèle et pour diverses raisons auxquelles s'ajoute sans doute l'éloignement géographique, il me semble qu'il y ait nettement moins d'échanges littéraires avec l'Afrique. On notera ainsi qu’à la différence de la France qui articule sa stratégie de "soft power" autour de ce type d'échanges dans l'ensemble de l'Afrique francophone, voire au-delà, le Canada semble vouloir continuer de miser sur une image éculée de "nation pacifique ». Un choix à contre-courant des aspirations exprimées par beaucoup d'Africains, de plus en plus nombreux à élever la voix contre les compagnies minières canadiennes qui seraient, du point de vue de l'éthique des affaires, tout sauf des parangons de vertu sur leur continent. Rares sont donc, à ma connaissance, les initiatives menées de part et d'autre en vue d'accroître les échanges 
entre acteurs canadiens et africains de la chaîne du livre. Je ne veux pas croire que le difficile accès au visa canadien, pour ce qui est des Africains, soit l'obstacle principal à cette situation qui oblige ceux qui déplorent le statu quo, à réfléchir aux voies et moyens de jeter des ponts entre Halifax et Casablanca, Moncton et Goma, Bamako et Sherbrooke.

J. J. : Aux ateliers de Dakar, on a longuement parlé de la question de la restitution des œuvres d'art. Considérez-vous que la littérature a, elle aussi, un rôle à jouer dans ce débat?

B. N. : La littérature est par essence le creuset autant de ce qui est, que et de ce qui pourrait être. Or la question que vous évoquez est bel et bien débattue depuis des années et bien avant les Ateliers de Dakar qui ont, j'en conviens, permis dans une large mesure de l'inscrire à l'agenda de certains pays d'Europe et d'Afrique. Si je me méfie par principe de toute forme que pourrait revêtir ce que j'appellerais l'écriture par injonction, je considère qu'il n'y a rien de plus naturel que de voir la littérature entrer en résonnance avec les débats qui traversent la société. Qu'elle précède de tels débats et en devienne le catalyseur ou qu'elle les enrichisse, idéalement en bousculant les certitudes, la littérature a toujours su étendre ses tentacules partout où les humains tissent les mailles de leurs destins individuel et collectif. La question de la restitution des œuvres d'art ne saurait y échapper.

\section{INDEX}

Mots-clés : francophonie, littérature congolaise, littérature canadienne, carrière d'écrivain, restitution des œuvres d'art, world literature

\section{AUTEUR}

\section{BLAISE NDALA}

Auteur de J'irai danser sur la tombe de Senghor et de Sans capote ni kalachnikov 